\title{
For Baby's Sake: Intervention Development and Evaluation Design of a Whole-Family Perinatal Intervention to Break the Cycle of Domestic Abuse
}

\author{
Jill Domoney ${ }^{1}$ (D) Elaine Fulton ${ }^{2} \cdot$ Nicky Stanley $^{3} \cdot$ Amanda Mclntyre $^{2} \cdot$ Margaret Heslin $^{4} \cdot$ Sarah Byford $^{4}$. \\ Debra Bick $^{5} \cdot$ Paul Ramchandani $^{6} \cdot$ Harriet MacMillan $^{7} \cdot$ Louise M. Howard $^{1} \cdot$ Kylee Trevillion ${ }^{1}$
}

Published online: 25 January 2019

(C) The Author(s) 2019

\begin{abstract}
For Baby's Sake is an innovative whole-family intervention that works with parents from pregnancy to two years postpartum to break cycles of domestic abuse and improve outcomes for children. The programme launched in 2015 across two community settings in England, with an independent evaluation led by King's College London. This paper aims to (1) summarise the process of developing For Baby's Sake and how it has been embedded within two different settings and (2) describe the evaluation design using early data to illustrate successes and challenges. The programme was developed following a review of the evidence and extensive stakeholder engagement. Three experts co-designed the content in partnership with the Stefanou Foundation and the programme delivery teams have been integrated into two local authorities. The evaluation uses mixed methods to assess abuse victimisation/perpetration, mental health, parenting and child outcomes, alongside service user experiences of early engagement. Forty individuals (27 women and 13 men) have been recruited to the evaluation. Early findings suggest that parents value the novel approach of For Baby's Sake and their relationships with practitioners. Data on parents' mental health and childhood adversities supports the decision to create a traumainformed intervention. Interventions for domestic abuse are necessary to improve health and behaviour outcomes for families and prevent intergenerational transmission of abuse and developmental trauma. For Baby's Sake addresses limitations of existing interventions, through its trauma-informed, attachment-based, whole-family approach. Early data from the evaluation suggests that the programme is reaching its intended audience and that service users appreciate the supportive approach.
\end{abstract}

Keywords Domestic abuse $\cdot$ Psychological treatment $\cdot$ Trauma $\cdot$ Intervention

Jill Domoney

jill.domoney@kcl.ac.uk

1 Section of Women's Mental Health, Health Service and Population Research Department, Institute of Psychiatry, Psychology and Neuroscience, King's College London, London, UK

2 The Stefanou Foundation, Welwyn Garden City, Hertfordshire, UK

3 School of Social Work, Care and Community, University of Central Lancashire, Preston, UK

4 King's Health Economics, Health Service and Population Research Department, Institute of Psychiatry, Psychology and Neuroscience, King's College London, London, UK

5 Florence Nightingale School of Nursing and Midwifery, King's College London, London, UK

6 Faculty of Education, University of Cambridge, Cambridge, UK

7 Departments of Psychiatry \& Behavioural Neurosciences, and of Pediatrics, Offord Centre for Child Studies, McMaster University, Hamilton, ON, Canada

\section{Introduction}

Around one in five children in the UK experience domestic abuse during their childhood (Radford et al. 2011) and this can lead to impairment across a range of health, social and developmental outcomes (Hughes et al. 2017). In particular, Research has shown that exposure to domestic abuse in the critical first 1001 days of life, from conception to the age of two, is associated with adverse outcomes throughout childhood and adolescence (Flach et al. 2011; Norman et al. 2012), including poor mental and physical health, lower academic achievement, and impaired social development (BairMerritt et al. 2006; Evans et al. 2008; Kitzmann et al. 2003). The impact of adverse experiences begins during pregnancy as the mother's emotional state can have a direct influence on fetal development by altering the environment in the womb (Glover and Capron 2017). Ongoing stressors can further disrupt the child's neurodevelopment, impacting on cognitive functioning and emotional regulation, which affect later 
emotional and behavioural outcomes (NSCDC 2007). Research has also identified early attachment as the foundation upon which future childhood, adolescent and adult relationships are formed (Moore et al. 2017). This hinges on sensitive, attuned caregiving by the parents particularly during the first 1001 days. Domestic abuse can undermine parents' ability to provide the consistent, sensitive and responsive caregiving that babies and young children need, especially if the parents did not receive this level of caregiving when they were children (Barlow and Underdown 2017). Indeed, a key mechanism through which the intergenerational transmission of risk is thought to function is parenting behaviour and, in particular, parent-infant interaction (Stein et al. 2014).

Adverse childhood experiences (ACEs) are common among adults reporting domestic abuse victimisation and perpetration (Coid et al. 2001; Ehrensaft et al. 2003). ACEs are stressful or traumatic experiences occurring in childhood and include physical, sexual and emotional abuse, physical and emotional neglect and living in a household with domestic abuse, among others. A seminal paper by Felitti et al. (1998), comprising a survey of 13,494 US adult healthcare attenders, found that those who had experienced 4 or more ACEs were at significantly greater risk of developing mental and physical health problems in adulthood. Since then many other studies have gone on to replicate these findings and look at additional outcomes related to ACEs (Dube et al. 2003; Edwards et al. 2003; Nusslock and Miller 2016; Reuben et al. 2016). Moreover, a 2017 systematic review and metaanalysis (Hughes et al. 2017) confirmed associations identified in the Felitti et al. paper and revealed strong associations between multiple ACEs and subsequent victimisation and perpetration of interpersonal abuse (including intimate partner abuse) in adulthood. The review found the odds of interpersonal abuse victimisation/perpetration in adulthood was seven times greater among those with $\geq 4$ ACEs compared to those without any ACEs. This review excluded high-risk groups and clinical samples but evidence suggests these groups are at increased risk of experiencing multiple types of interpersonal violence victimisation across the lifetime (Anderson et al. 2016; Clements-Nolle et al. 2009; Khalifeh et al. 2013, 2015). ACEs have also been shown to be associated with subsequent parenting behaviours, perceived parenting stress and perceived child development, although maternal mental health problems are seen to mediate these associations (Guss et al. 2018; Sun et al. 2017).

There is evidence of strong associations between domestic abuse victimisation/perpetration and a wide range of mental disorders, including depression, post-traumatic stress disorder, sleep and eating disorders, suicidal behaviour and misuse of alcohol and drugs (Devries et al. 2014; Howard et al. 2013; Riggs et al. 2000; Trevillion et al. 2012). Mental health problems may arise as a direct result of experiencing domestic abuse when conceptualized in a trauma framework, with evidence that the extent and severity of abuse experienced is strongly linked to the severity of symptoms of mental disorders (Golding 1999; Trevillion et al. 2012). In addition, pre-existing mental health problems can impact on people's vulnerability to domestic abuse, and therefore associations may represent bidirectional effects (Trevillion et al. 2012). For those who use abusive behaviours, experience of childhood abuse is common, and this is a risk factor for both adult mental illness and domestic violence perpetration (Machisa et al. 2016).

To date, domestic abuse interventions have tended to target either perpetrators alone or women and/or their children; there are few programmes that aim to provide integrated treatments to the whole family and even fewer which address the mental health difficulties of parents. There have been some interventions piloted in the UK that aim to address perpetrators' parenting (McConnell et al. 2017) and some perpetrator programmes also deliver support services to participants' partners and children (Alderson et al. 2013). However, the majority of interventions which address the impacts of domestic violence on families concentrate on those with school-aged children; there are only a small number of programmes that work with families during the perinatal period (Jack et al. 2012; Jahanfar et al. 2014), and these predominantly work with mothers and children alone.

It is increasingly acknowledged that separation or a cessation of contact between the abusive parent and their children are not always the safest or the preferred solutions for families living with domestic abuse (Stanley 2011) and as a result a few examples of 'whole family' interventions are emerging that aim to work with all family members whether they live together or not. These approaches reflect the shift towards increasing perpetrators' accountability regarding the impacts of children's exposure to domestic abuse and the fact that research suggests fatherhood may be a significant motivator for behaviour change (Meyer 2018; Stanley et al. 2012b). Stanley and Humphreys describe a range of models and programmes from Europe, Australia and the USA that vary considerably in their length and are delivered from very different settings (Stanley and Humphreys 2017). However, at this point in time, these interventions do not provide a holistic package of support which includes wholefamily work, early therapeutic intervention, infant development and parental mental health.

For Baby's Sake is the first to address existing limitations of whole-family interventions, as it combines evidence-based treatments for domestic abuse, trauma and adult mental health, alongside parenting interventions focused on infant mental health and parent-infant attachment. It also includes support for parents to overcome the impact of their own childhood trauma. Delivered through pregnancy until the child's second year, trained practitioners work separately with expectant mothers and fathers with the goals of breaking cycles of 
domestic abuse and ensuring the best outcomes for children. For Baby's Sake aims to engage each family member through a single, synchronised programme that coordinates each person's involvement, enabling a full picture of the risks and potential impacts for all involved.

The aim of this paper is threefold: (1) to summarise the process of development of For Baby's Sake and how it has been embedded within two different regional community settings, (2) to describe novel methods used to measure service user outcomes and (3) to present data that illustrates early successes and challenges of conducting an evaluation of a complex, whole-family intervention.

\section{Section One: The Process of Development and Implementation of For Baby's Sake}

Informed by current research, consultation and input from three expert co-designers, For Baby's Sake aims to intervene with expectant parents: (1) to bring an end to domestic abuse; (2) to overcome the impact of traumatic experiences in their own childhoods; (3) to make sustained changes in their behaviour, and (4) to create the conditions through their parenting techniques and family environment to prevent poor outcomes for infants. For Baby's Sake launched in spring 2015 across two different community settings and regions in England, with an independent mixed-methods evaluation led by King's College London which is outlined in section two.

\section{Creating For Baby's Sake}

\section{Scoping of the Programme}

The Stefanou Foundation, established as a philanthropic charity in 2008, has developed its model of catalytic philanthropy aiming to transform the lives of those most at risk and unable to tell, particularly among the very young.

Work on developing For Baby's Sake took place over five years and included a detailed review of the evidence-base as well as extensive stakeholder engagement to shape the intervention.

It was clear early on that any new programme needed to be integrated with public services and established safeguarding regimes. The Stefanou Foundation, therefore, initiated preliminary work in two Local Authority areas within London and Hertfordshire.

The two prototype sites were selected partly because of their different populations, urbanicity, and different public sector structures, in order to test the acceptability and functioning of the programme in different contexts. One site covers an area within the county of Hertfordshire which has a population of approximately 1.18 million people, of which $81 \%$ are white British, $62 \%$ are of working age and
$32 \%$ have a level 4 qualification (i.e. university degree, higher education or professional qualification); in the year 2017 there were a total of 14,301 live births to mothers residing in the county (Office for National Statistics 2016, 2018). Communities in this area are relatively stable, with successive generations often living close to each other. By contrast, the London site, which originally covered one London borough and expanded into two further boroughs, is a highly diverse and transient community, with a population of approximately 560,538 people, of which less than $50 \%$ are white British, around $70 \%$ are of working age and around $50 \%$ have a level 4 qualification; in the year 2017 there were a total of 6572 live births to mothers residing in the tri-borough. Local government, health and policing structures also differ between the two sites, with different practical implications for building referral pathways and multi-agency arrangements for safeguarding and securing support from partner agencies to meet service users' complex needs, including access to mental health, drug and alcohol and housing support. For example, the Hertfordshire families participating in For Baby's Sake overwhelmingly access maternity services from a single hospital, whereas the London families are using several hospitals across the capital.

Building on existing relationships within these two areas, the Stefanou Foundation convened professionals from local government, health, police and probation to help shape the intervention that the Local Authorities would subsequently host. Mechanisms included creating a Steering Group which brought together senior representatives from statutory agencies across the two Local Authority areas along with a few national experts in the fields of domestic abuse, parenting and public policies related to social justice and early intervention. This proved particularly valuable in informing the elements of the programme's design and narrative which needed to reconcile pressures from different organisational cultures and disciplines, while holding true to the Stefanou Foundation's evidence-based theory of change for the programme. Research, consultation and networking also took place with expert practitioners, academics and campaigners in the fields of domestic abuse, infant mental health, family support and early intervention. These activities continued throughout the design phase to keep abreast of the evidence that was emerging over this period and to build understanding and trust in the programme's innovation and in the Stefanou Foundation's ability to deliver it. The Steering Group's approval of a set of core characteristics of the programme, in 2012, was a pivotal moment in terms of securing senior stakeholder support for the proposed model. These core characteristics openly articulated some key imperatives and the tensions to be managed, for instance the imperative to prioritise safety while also creating the conditions for change; and to form therapeutic alliances with service users that were motivational, non-judgmental and strengths-based, whilst remaining non-collusive and realistic. 


\section{Design of the Therapeutic Programme}

The Stefanou Foundation identified three experts who worked within the different strands that the programme intended to address: Dr. Roxane Agnew-Davies (expert in addressing domestic abuse and its impact on women's mental health); Mark Coulter (expert in supporting men to bring an end to perpetration of domestic abuse); and Dr. Christine Puckering (expert in sensitive, attachment-based parenting). The task given to these experts was not to 'glue together' existing programmes for domestic abuse victims/survivors, domestic abuse perpetrators and vulnerable parents and babies. Rather, the brief was to work closely with the Stefanou Foundation to create a new, holistic and carefully synchronised programme, which would integrate support for each family member (mother, father, baby, young siblings) and prioritise mental health and parent-child attachments.

The Stefanou Foundation facilitated a learning exchange between the co-designers and invested considerably in bringing them together with time committed to understand each other's expertise, approaches and influences. Notably, an ecological framework (Heise 1998) captured factors that set the stage for domestic abuse and highlighted the need to address variables at different levels of the system. Key lessons were learnt about the help-seeking behaviours of abusive men (Stanley et al. 2012a). The Bakermans-Kranenburg meta-analysis of what works in infant mental health, notably work on sensitivity and use of video, helped to shape the content on attachment-based parenting support (Bakermans-Kranenburg et al. 2003).

Early discussions identified common therapeutic themes at the core of the co-designers' different areas of work, including reclaiming the inner child and the healthy expression of emotions including anger, grief, shame and fear. These themes became central to the therapeutic model. Techniques were also shared and adapted for inclusion in the programme around: (1) harnessing the help-seeking behaviours of the programme participants/users; (2) creating a therapeutic alliance; (3) avoiding stigma; (4) building self-esteem, and (5) combining robust risk management with facilitating change and development.

The programme timeline for families was driven above all by the evolving needs of the baby from conception to age two. For example, reducing stress is a priority in all programme activity that takes place before birth, given the impact of sustained high levels of stress on babies in the womb (Glover and Capron 2017).

\section{Service User Input to the programme's Design and Development}

Service user voices have helped to inform the design and development of the programme at various stages. Early opportunities to hear from first time teenage parents participating in the Family Nurse Partnership (Jack et al. 2012) (where there was a high prevalence of domestic abuse) arose through the Stefanou Foundation's participation in multiagency forums. A specific meeting was arranged with men who had participated in the Strength to Change programme, which had been designed following a social marketing research initiative to understand the help seeking behaviours of abusive men (Stanley et al. 2012a).

In 2014 the Stefanou Foundation commissioned research from the National Social Marketing Centre (NSMC; a centre of excellence for social marketing and behaviour change based in the UK) to inform the development of the programme and any marketing messages. Interviews and focus groups were held with 17 stakeholders including eight mothers who had experienced domestic abuse and one father who had perpetrated domestic abuse. Their reflections provided valuable insights into the complex mix of emotions for expectant mothers and fathers, which informed the programme's approach to harnessing parents' motivations and being sensitive to their fears about seeking help at this time. Most of the mothers interviewed had asked their partners to seek help to address their abusive behaviours, indicating that the whole family approach of For Baby's Sake would be welcomed. Parents also reported having struggled to find appropriate support for themselves and their partners. Those who most valued the support they had received emphasised the consistency and therapeutic approach of the person working with them. Stakeholders, survivors and perpetrators highlighted the lack of support for the psychological impact of domestic abuse and for the wider emotional and mental health needs of many domestic abuse survivors and perpetrators. They saw For Baby's Sake as having the potential to fill that gap.

The programme was launched in 2015 with a working title of Healthy Relationships: Healthy Baby (HRHB). Feedback from interviews with stakeholders and service users highlighted some confusion arising from the programme's working title that it sought to address parental relationships and/or parenting practice only. Its meaning was lost further by the tendency for the long name to be abbreviated into an acronym. In 2017, the Stefanou Foundation worked with a marketing and branding expert to identify a permanent name and associated branding for the programme. Feedback had underlined how the programme was harnessing parents' motives to give their babies a better start than they had experienced, and therefore the programme was named For Baby's Sake. Feedback from parents was also instrumental in shaping the language, messaging and tone of the new marketing materials.

Throughout the delivery of the prototype projects since 2015, For Baby's Sake has drawn on qualitative and quantitative data and feedback from practitioners and service users to inform the ongoing development of the programme. One such development was the decision to deliver the manualised 
sessions around healthy expression of emotions earlier in the programme. These sessions focus on handling feelings of guilt and shame and understanding dissociation, which were identified as important in improving parents' ability to discuss their feelings so that they could access fully the therapeutic content of the programme related to their own childhood trauma.

\section{Description of the Programme}

For Baby's Sake has been developed as a structured, modular programme which is delivered flexibly to meet individual needs. Staff work for up to two and a half years with expectant mothers and fathers as co-parents, whether or not they are together or stay together as a couple. Using a strengthsbased model, they work separately but in a coordinated way with both the mother and father to address their complex issues and support lasting behaviour change, alongside managing the risks for each family member, and acting swiftly to address any safeguarding concerns that may emerge. Staff delivering the programme come from a variety of professional backgrounds, including, for example, police, probation, the domestic violence sector, and early years' services. Prior to working with families they undertake a significant amount of training which covers topics such as safeguarding, mental illness, parent-infant relationships, and therapeutic skills, alongside specific training on delivering the intervention manual.

Therapeutic sessions are delivered face-to-face and are designed to assist parents to face up to past behaviours and experiences, including adverse childhood experiences, and current domestic abuse. Trauma experts such as Bessel van der Kolk, who have been advocating for complex developmental trauma to be recognised as a diagnosis, highlight that traumatic childhood adversity histories are associated with diagnoses such as personality disorders in adulthood, and note the difference between a diagnosis of PTSD (often rooted in an isolated traumatic experience) and the impact of repeated exposure to traumatic experiences (Van der Kolk 2015, 2017). The nature of this impact points towards the need for interventions that enable emotional self-regulation, take a traumainformed approach to breaking behaviour patterns, and support parenting behaviours which promote secure attachment in the children of traumatised parents. There is therefore an emphasis on empowering parents with life skills to reduce stress and maintain healthy adult relationships, and both parents are supported to give their babies and children the consistent and sensitive care that leads to secure attachments (Lucassen et al. 2011).

The programme integrates a range of therapeutic techniques to support behaviour change and recovery from trauma, including Cognitive Behavioural Therapy (Beck 2011), Transactional Analysis (Berne 2016) Gestalt techniques (Kellogg 2014), mindfulness (Whitaker et al. 2014) and systemic practice in Motivational Interviewing (Rollnick and Miller 1995). The Inner Child work (Bradshaw 1992) is at the therapeutic core of the programme in enabling parents to come to terms with their adverse childhood experiences and recover from trauma. The content helps parents to explore how their behaviours, thoughts and feelings may be being replayed from or triggered by their past. By being helped to recognise, connect with and look after the child within themselves, they are supported to build internal resilience for themselves and capacity to be a 'good enough' parent to their baby. This can include an acceptance of how their needs were not met during childhood and also drawing on the strength that originated from places or people that made them feel safe, loved or heard as children. The therapeutic relationship between service users and practitioners is crucial to engagement throughout the programme and especially to the Inner Child module. The programme's content and approach to working with parents are designed in order for mothers and fathers to be confident that For Baby's Sake will not judge them for their behaviour or what they have experienced, but will require, and empower, them to take responsibility for their own lives and for their baby's emotional, social and physical development.

The therapeutic and attachment-based parenting work provided for both mothers and fathers includes use of the Brazelton Newborn Behavioural Observation (Nugent 2015) and Video Interaction Guidance (Kennedy et al. 2011). These are tools which aim to help sensitise parents to their baby's communications and competencies and foster positive parentinfant interactions.

\section{Preliminary Work on Implementation}

At the heart of the Stefanou Foundation's operating model and the design of For Baby's Sake is full integration within local systems and processes, including co-location with local services within a children's centre in London and with a 'Troubled Families' team in Hertfordshire (a UK programme of targeted intervention for families with multiple problems), and use of the local authority case management systems. This ensures appropriate information sharing with children's social care and other colleagues, delivering a comprehensive picture of individuals' needs and risks in order to safeguard children and adults at risk. It also brings learning for everyone involved and supports wider service and system change and development.

The Stefanou Foundation's senior leadership team led the operational elements of the programme's development and negotiations to ensure that it would be integrated with key public services and safeguarding regimes. Key elements included: (1) designing team structures to offer each family member a therapeutic alliance and safe case management; (2) designing staff roles, recruiting and inducting them; (3) 
negotiating co-location arrangements with host local authorities and broader arrangements to achieve embedded working with them; (4) defining the programme's cohort inclusion criteria; (5) identifying key outcomes and indicators for the programme, and (6) commissioning the external research evaluation, led by King's College London. In addition, a range of programme marketing materials were developed for both professionals and families in order to provide information about the programme and how to make referrals.

Following this extensive development work, For Baby's Sake began working with families in the two Local Authority areas in different regions of England in 2015. The programme accepts those who:

- Are expecting a baby and ideally have not reached 28 weeks of pregnancy

- Are experiencing domestic abuse within their relationship, where the father is the main perpetrator of the abuse

- Wish to share the parenting of the baby, whether or not they are/stay together as a couple

- Will both be aged over 17 years when the baby is born

In addition to commissioning the external evaluation described below, the For Baby's Sake teams collect data on the characteristics and progress of the participants as well as process data about how the programme is operating. This data set is used for operational management, including safeguarding, and to produce reports to keep partners abreast of progress and maintain their engagement in supporting the delivery and development of the programme.

\section{Section Two: a Critical Review of Evaluating Service User Outcomes}

The choice of methods for the evaluation of For Baby's Sake took into account a number of factors: aligning with the underlying theory of change by measuring variables which the programme aimed to impact on (e.g. parenting, child developmental outcomes) (De Silva et al. 2014); using a mixed methods approach to capture a detailed and complete picture of service users' experiences and outcomes (O'Cathain et al. 2007); and balancing detailed assessments with participant burden. At this early stage of programme evaluation, a comparison group was not included and so a full clinical and economic effectiveness of the programme was not possible. Rather, the evaluation aimed to examine factors relating to the successful implementation of the programme within the two areas and provide early indications of the potential for beneficial clinical and economic outcomes for service users.

This section focuses on describing the methods used to measure service-user outcomes in order to highlight some of the successes and challenges in selecting measures which provide sufficiently detailed data on the outcomes of families participating in a complex, whole-family intervention. This is followed by presenting some of the early findings from the evaluation of the programme, illustrating how these have been used to inform ongoing refinements to both the evaluation and the programme.

Evaluation is ongoing at the time of writing and is due to be completed in the summer of 2019.

\section{Research Interviews with Service Users}

Recruitment to the evaluation ran from June 2016 up until the end of July 2017. During the sign-up process to the programme, For Baby's Sake staff asked family members for consent to be contacted by the evaluation team. Those who consented to take part in the evaluation are visited at three time points: at initial sign-up and again at approximately one year and two years into the programme. The face-to-face research interviews comprise self-report and researcher-administered questionnaires, a qualitative interview and, postnatally, observed measures of child development and parenting.

Prioritising participant safety is an essential feature of these research interviews and the evaluation team have developed detailed standard operating procedures and safety protocols. These include clear guidelines around how to contact potential participants, flexibility in the location of interviews to ensure privacy, and ongoing discussions with participants about potential risks and how to mitigate them (Ellsberg and Heise 2002; WHO 2001). In particular, males and females are treated as entirely separate participants, are interviewed separately in a private setting which cannot be overheard and are not given information about each other's interviews.

\section{Selecting Measures}

Domestic Abuse Given the focus of the programme, assessments of domestic abuse perpetration and victimisation are key measures in the evaluation. Research has shown that self-reports of abuse victimisation are the criterion standard for assessing exposure (MacMillan et al. 2009), and partner-reports of abuse perpetration increase reliability and validity of assessments of exposure (Hester et al. 2015). However, some existing self-report questionnaires which are commonly used (e.g. the Conflicts Tactics Scale) have been criticised for reducing item questions to simple behaviour-based checklists which focus on acts of noninjurious physical aggression and which do not collect data on the abuse incidents or impacts (Hamby 2016; Loseke and Kurz 2005). Robust measures need to capture not only the different types of abuse, but also the frequency and severity of abuse, as well as the impact it has on different domains of functioning.

The evaluation team took into consideration the issues with existing measures in developing a questionnaire which draws on previously tested/gold-standard questionnaires that have been used with similar population groups (Hegarty et al. 
2005; Hester et al. 2015) and includes measures of changes in frequency, severity and impacts of abuse over the course of the programme. In addition, some items that measure the number of abusive incidents and whether these incidents resulted in criminal justice contacts have been included. The abuse measure assesses a range of physical and non-physical forms of abuse - i.e. physical, sexual, psychological, emotional and financial abuse - including forms of controlling and coercive behaviours. The Composite Abuse Scale (Hegarty et al. 2005) is also used to capture more detailed information on current domestic abuse victimisation; this measure has been used successfully with women and men in clinical populations (Trevillion et al. 2013).

Mental Health Assessment of a range of mental health disorders and symptoms was included to help identify potential causal mechanisms and contextual factors which may explain variations in outcomes. This included screening measures for symptoms of anxiety (Generalised Anxiety Disorder Assessment (GAD-7) (Löwe et al. 2008)), depression (Edinburgh Postnatal Depression Scale, (Cox et al. 1987)), post-traumatic stress disorder (PTSD: Posttraumatic Diagnostic Scale (Foa et al. 1997)), personality disorder (The Standardised Assessment of Personality Abbreviated Scale (SAPAS) (Moran et al. 2003)), and drug and alcohol use (the Alcohol Use Disorders Identification Test (Babor et al. 2008) and the Drug Use Disorder Identification Test (Berman et al. 2003)).

Parenting and Infant Outcomes The programme specifically aims to improve the parenting behaviours of both mothers and fathers in order to promote healthy development of infants and includes interventions such as Video Interaction Guidance (Kennedy et al. 2017) which targets parent-infant interactions directly.

To identify the most suitable methods for measuring child outcomes and parenting in this population, it is necessary to draw on domestic abuse and perinatal mental health evidence. Presently, most domestic abuse research on adverse parenting and child outcomes focuses only on mothers' self-reported perceptions (Huth-Bocks and Hughes 2008; Johnson and Lieberman 2007; Letourneau et al. 2007). This approach fails to address potential reporting biases. Indeed, a study comparing researcher-observed mother-child interactions against mothers' self-reports found different results in relation to the impacts of abuse on children's behaviour (Levendosky et al. 2003). Traditionally, the domestic abuse literature has also failed to recognise children as active agents who play a role in shaping the mother-child relationship (Katz 2015; Överlien 2017). There has also been little emphasis on development and use of valid and reliable assessments for fathers (Labarre et al. 2016; Rothman et al. 2007; Salisbury et al. 2009) and, where utilised, these are often self-report (Stover et al. 2013).
These limitations are notable as domestic abuse is found to have differential impacts on parenting attitudes and practices among mothers and fathers (Margolin and Gordis 2003).

The perinatal mental health literature can address some of these limitations; objective measures of parent-infant interaction are commonly used in this field, primarily through the use of observed/video-taped parent-infant interaction which are coded using constructs such as parental sensitivity, mindmindedness, and dyadic synchrony. These kinds of assessments acknowledge children as active agents in the relationship. The constructs they measure have been found to mediate the association between parental psychiatric disorders and child outcomes (Lyons Ruth and Block 1996; Murray and Cooper 1996) and, importantly, are predictors of outcomes for both mothers and fathers (Lucassen et al. 2011). The importance of measuring both parents' interactions with their child is also highlighted in the perinatal literature as the sensitivity of one parent may 'buffer' the lower sensitivity of the other (Malmberg et al. 2016). In addition, research into the impact of parental mental illness on child outcomes has highlighted the need to measure a range of domains of child functioning (e.g. behavioural, emotional, and cognitive), as these may be differentially affected by deficits in parenting (Murray et al. 2015).

To address the above limitations, the evaluation employs a combination of observed and parent-report child measures across several domains of functioning. This includes using standardised measures such as the Bayley Scales of Infant Development (Bayley 2006), the Infant Behaviour Questionnaire (Gartstein and Rothbart 2003) and the Child Behaviour Checklist (Achenbach and Edelbrock 1983) to examine cognition, language, temperament and internalising and externalising behaviour. In terms of attachment, a parentreport measure of prenatal bonding is used at baseline, and the feasibility of administering the gold-standard Strange Situation procedure at the two-year visit has been assessed.

The CARE Index (Crittenden 1979) is used to assess the quality of parent-infant interaction for mothers and fathers. Importantly, it measures the relationship between infant and caregiver, seeing infants as active agents (Överlien 2017), and therefore captures the dynamic and reciprocal nature of interactions.

\section{Participant Experience of the Programme}

In addition to the standardised measures described above, qualitative interviews are being undertaken with participants at the three time points: baseline interviews include questions about the reasons for referral and individuals' expectations of the support provided by the programme; at later time points, participants are asked to consider whether the programme is meeting their expectations and to identify any aspects of the content that they have found particularly helpful. They are 
also asked to reflect on any changes that they have noticed and their beliefs about what has led to change. This data is essential to understand the experience of those undergoing the programme and to provide suggestions for potential adaptations and refinements in the future. Such information can also be integrated with the quantitative data to provide insights into mechanisms of change, as well as potentially capturing unanticipated causal pathways (O'Cathain et al. 2007).

\section{Early Data on Engagement and Experiences of Service Users}

Early data from service-users has been used to assess the feasibility of the evaluation methods, to explore the characteristics of those attracted to the programme and to find out about service users' experiences of engaging. These findings are used to feed into ongoing refinements to both the evaluation and the programme. Here we present some preliminary data from baseline interviews with participants, detailing the ways in which this has been used to adapt and refine the evaluation to adequately meet service user needs, as well as how it has contributed to ongoing programme development. The evaluation is due to be completed in summer 2019, at which time further results will be published.

\section{Feasibility of Administering Measures}

During early interviews, the feasibility of administering the full baseline interview schedule was piloted. This included recording the time taken to complete measures, recording any items which presented challenges to administer and asking for verbal feedback from participants about their experience of the interview. Based on this, several changes were made to the participant interview schedule early in the data collection process to reduce burden and improve acceptability.

First, the interview schedules were organised into two sections - one that lists essential measures that the interview should cover and one that lists desirable measures that the interview could cover if participants do not feel overburdened by the interview procedures.

Second, the self-complete diagnostic clinical interview the Clinical Interview Scale-Revised - CIS-R (Lewis et al. 1992) was removed. This assessment could take up to $15 \mathrm{~min}$ to complete and was burdensome for participants given that a range of mental health screening tools are also administered, some of which capture the same disorder constructs as the diagnostic interview. Although it is not possible to determine whether participants met the clinical threshold for a mental disorder, symptoms of depression, anxiety, posttraumatic stress disorder, personality disorder, and use of alcohol and drugs continue to be assessed through validated screening questionnaires.
Third, the Strange Situation (Ainsworth and Bell 1970) was omitted from the evaluation. This is a laboratory-based structured observation, which is designed to assess the attachment relationship between a child and caregiver. This was intended to be completed at the 2-year follow-up interview. However, most families in the programme are engaged with a range of services so have multiple competing commitments, and several fathers in the evaluation have restricted contact with the infant. In addition, families would need to travel long distances to attend a laboratory where this procedure could be undertaken. Therefore, the likelihood of obtaining data from enough families to draw any meaningful conclusions about this outcome is very low and so a decision was taken to omit this measure.

Finally, some changes were made to video assessments. To undertake the CARE Index assessments, families are asked to agree to being filmed while playing with their infants. Being video-taped is a challenge for this group; as well as typical barriers such as embarrassment about being filmed and uncertainty about who will see the videos, some participants do not live with their children or have restricted access. In order to overcome this, the evaluators sought consent from participants to use videos that were taken as part of the intervention (i.e. Video Interaction Guidance). This method has provided baseline data from a wider group of individuals and, especially, fathers.

\section{Quantitative Data}

Between March 2015 and March 2017, the programme received 245 referrals. These came primarily from social services and midwifery, but also from other sources such as police, mental health services and self-referrals. Just under $80 \%$ of these referrals met the criteria for the programme. This information was used to target services who were not currently making referrals and improve referrers' understanding of the programme criteria in order to reduce inappropriate referrals.

During recruitment, the contact details of a total of 88 individuals who provided consent for contact were received by the evaluation team across both sites. This was approximately one third of those referred to the programme. Baseline interviews were completed with 40 individuals $(45 \%)-27$ women and 13 men. Reasons for non-completion included withdrawal of consent $(n=11)$, baseline period passed before an interview could be arranged $(n=4)$, persistent non-response to attempts at contact $(n=12)$, withdrawal from the programme and unable to contact/declined baseline $(n=21)$.. The noncompletion rate highlights the challenges with recruiting participants to an evaluation. Multiple efforts were made by the evaluation team to engage with those who dropped out or did not engage following referral to the programme. As well as attempting to make contact several times across a range of 
platforms, a brief, anonymised electronic survey was developed, with incentives for participation (i.e. a $£ 5$ coffee voucher for disclosing the reason for non-engagement). The survey was developed to help address potential barriers to participation, but this had limited success. Nevertheless, the number of individuals recruited is similar to other published pilot and prototype interventions for those who have experienced and perpetrated domestic abuse (e.g. Constantino et al. 2005; Schumacher et al. 2011).

In terms of sample characteristics, women $(n=27)$ who completed baseline interviews had a mean age of 28 (sd 7.5). $75 \%$ were White British and $85 \%$ had completed education beyond age 16. $37 \%$ were married or cohabiting and $40 \%$ were first time parents. Nearly a third (29\%) were currently smoking and just under half (44\%) reported they had a mental illness.

Men $(n=13)$ had a mean age of 29 (sd 7.7). 85\% were White British and 55\% had completed education beyond age 16. In term of their relationships, $54 \%$ were married or cohabiting and $38 \%$ were first time parents. Over three quarters (77\%) were currently smoking and over half (54\%) reported they had a mental illness.

All families were assessed as currently experiencing domestic abuse at the point of sign-up to the programme.

Screening measures of mental illness indicated that, at baseline, $40 \%$ of women had symptoms of anxiety, $45 \%$ had symptoms of depression, $18 \%$ had symptoms of PTSD and 54\% had disordered personality traits. For men, $46 \%$ had symptoms of anxiety, $38 \%$ had symptoms of depression, $30 \%$ had symptoms of PTSD and $77 \%$ disordered personality traits.

The high levels of mental health difficulties in the sample is in line with literature on the association between mental health and experience of domestic abuse (Howard et al. 2013). This also reinforces the need for a therapeutic programme to provide psychological support for victims and perpetrators, which was highlighted in the NCSM report commissioned by the Stefanou Foundation to inform the programme development, and subsequently incorporated as a key feature of the programme. Similarly, the many participants that are reporting PTSD symptoms and disordered personality traits suggests that the programme is reaching parents with trauma histories, providing support for the trauma-informed approach adopted by For Baby's Sake.

\section{Qualitative Data}

Analysis of baseline qualitative interviews identified several themes relating to the motivations and reasons that service users signed up to the programme. Many women described poor experiences of previous support and were attracted to the novel approach of For Baby's Sake. They particularly valued the supportive relationship with their practitioner: "It's quite nice to, I don't know, to have a relationship because they're not just your worker, you end up forming a bond, like a relationship with them, which is quite nice.”

Their motivations often focused on giving their new baby a good start in life, but also on a desire to get support for their relationship, with many women being pleased that their partners would be involved. As one woman said, "There have always been a lot of problems in the relationship. So to not treat it just through me, but actually have [male partner] being part of that, yes, I was really excited about that.”

Men also described histories of feeling judged and dismissed by other services and, similarly to women, their motivations for signing up centred on wanting to improve the relationship with their partner and provide a good start for their baby: "...to have that family around him [child] that I never had, that's the most important thing that I want him to have, the family that I wanted."

Some men were able to acknowledge abusive behaviours and expressed a desire to change, while others struggled with the language of abuse and perpetration but found that early positive contacts with practitioners helped them to engage. "He [practitioner] was talking with a great deal of confidence about his field and about previous experience in cases. He did a very good job building my confidence in him and in the programme."

Qualitative data from service users has corroborated the need for consistent, non-judgemental support which was highlighted by stakeholders in the early scoping work. Data from interviews has also fed back into adaptations to the programme. For example, some of the language used in the signup process, such as 'perpetrator' and 'victim', is no longer used in order to attract and retain people on the programme, and some of the manualised sessions related to the healthy expression of feelings have been brought forward to better meet service user needs.

\section{Discussion}

For Baby's Sake is an innovative whole-family intervention that works with parents from pregnancy to two years postpartum to break cycles of domestic abuse and improve outcomes for children. It aims to address existing limitations of wholefamily interventions, as it combines evidence-based treatments for domestic abuse, trauma and adult mental health, alongside parenting interventions focused on infant mental health and parent-infant attachment. The programme launched in 2015 across two community settings in England, with an independent evaluation led by King's College London. Here we discuss some of the issues arising from the early stages of programme implementation and evaluation.

For Baby's Sake aims to address the complex nature of abusive relationships by using a strengths-based, trauma- 
informed model and supporting parents to overcome their adverse childhood experiences and recover from trauma. The data on participants' mental health, collected by the evaluation team, not only highlights the associations between mental health and experiences of domestic abuse, but is also pertinent to the trauma-informed and attachment-based nature of For Baby's Sake. A high percentage of parents recruited to the study had disordered personality traits and PTSD symptoms. Trauma experts highlight that traumatic childhood histories are associated with diagnoses such as personality disorders in adulthood (Van der Kolk 2015, 2017). This sits alongside reports from For Baby's Sake practitioners of a high percentage of parents who have experienced four or more adverse childhood experiences, indicating that, as intended, For Baby's Sake is reaching parents with childhood trauma histories. The nature of this impact points towards the need for interventions that enable emotional self-regulation and take a trauma-informed approach to breaking behaviour patterns.

The programme is delivered flexibly to meet individual needs and staff work for up to two and a half years with expectant mothers and fathers as co-parents, whether or not they are together or stay together as a couple. The flexibility offered by the staff (e.g. in offering out-of-hours sessions, visiting families at home, offering face-to-face and telephone sessions) and consistent support were noted by service users as a key strength of the programme and something that aided engagement. A non-judgemental, strengths-based therapeutic alliance was highlighted as a core characteristic early in programme development, and this has been borne out by feedback from service users as a key component in supporting change.

The two prototype sites were chosen partly because of their different populations, urbanicity, and public sector structures. It is a strength to have this diversity within the prototype sites so that early lessons can be learned about implementation in different settings and potential differences in service user needs. For example, referral sources differed across the localities leading to different strategies by the two teams to increase referrals. However, the diversity also represents challenges for evaluating impact, as recruitment and retention is substantially lower where the population is more transient. This is also reflected in the data from the independent evaluation, whereby significantly more participants have been recruited and retained from one of the sites.

The choice of methods for an evaluation of a multifaceted, whole-family programme needs to consider a number of factors, including aligning measurement with the underlying theory of change (De Silva et al. 2014); using mixed methods that can capture different levels of experience (O'Cathain et al. 2007); and minimising participant burden. In the context of evaluating a wholefamily domestic abuse intervention, delivered in the perinatal period, there are several additional considerations. Valid and reliable tools are needed to measure experiences of abuse victimisation/perpetration, parenting and child outcomes, as these are key variables which will be used to understand the impact of the intervention. However, these tools also need to be feasible to deliver and acceptable to participants in a population with multiple, changing needs. The evaluators drew on measures from the fields of domestic violence, perinatal mental health and child development to provide a broad overview of the early impacts of For Baby's Sake and piloted the feasibility of administering these measures. The interview schedule was adapted in response to the pilot, while maintaining measures across key domains.

There were both successes and challenges in the early stages of the evaluation. It is recognised that there are significant challenges in engaging participants to take up domestic abuse interventions and additionally to participate in an evaluation (Stanley and Humphreys 2017). The rich participant data that is being collected as part of the evaluation is only available for a sample of the families in the programme (approximately one third consented to be contacted by the evaluation team, and just under half of these individuals engaged with the evaluation) The extent to which those participating in the evaluation share characteristics with those who either declined to be in the evaluation but did engage with the programme, or those who dropped out of the programme after initial engagement, is important to examine. This can be done to some degree by drawing on the data collected by the programme staff about the families in the programme. The evaluation team will be able to use an anonymised format of this data to attempt to answer questions about the representativeness of families in the evaluation, and to inform questions around the reach of the programme. This dataset will be informative to the evaluation when considering the generalisability of findings.

Additionally, the sample who are engaged with the evaluation includes significantly more women than men meaning that data cannot always be collected on all members of the household. Furthermore, where men have engaged, it is sometimes not possible to collect data on father-infant interactions if men have restricted access to their child. These experiences highlight the challenges of evaluating a wholefamily intervention from the perspective of all family members (Stanley and Humphreys 2017).

Similarly, multiple moves and relocations characterise the experience of families living with domestic abuse and the evaluators have found that many participants change their contact details and home addresses between time points, which can create challenges for ongoing engagement. To address this, the evaluation team uses strategies to maintain contact with families between interviews (e.g. 
sending Christmas and birthday cards) and programme staff are asked to assist in re-establishing contact with participants. This has helped to maintain engagement across the evaluation period.

Despite these challenges, early data suggests that the programme is able to reach parents with trauma histories and that the approach to intervention is appreciated by service users. The evaluators have successfully collected a range of mixed methods data from service users which are feeding back into programme development and will provide indications of the potential for beneficial outcomes for families.

\section{Conclusions}

Early interventions for domestic abuse are necessary to promote healthy outcomes for children and prevent intergenerational transmission of abuse and trauma. For Baby's Sake is novel in its approach to providing a whole-family intervention, delivered during the perinatal period and targeting domestic abuse, parent-infant attachment and addressing parents' own histories of trauma. While the evaluation described here is still ongoing, the experiences described above highlight that it is possible to implement a whole-family domestic abuse intervention within community settings, and to work intensively with mothers and fathers with the aim of improving outcomes for children living with domestic abuse. The evaluation draws together measurements from the fields of domestic abuse, perinatal mental health and early child development to provide a broad picture of the impacts of the programme. Collecting outcomes from transient populations and from all members of the household in order to get a full picture of impact poses challenges and requires flexible and novel approaches to measurement. Early data has been useful in refining the assessment schedule to reduce participant burden as well as feeding back into ongoing programme development. In particular, data from service user interviews has provided learnings about the language which can attract families to the programme, the importance of consistent, nonjudgemental support, and the need for flexible delivery with this population. The evaluation continues until the summer of 2019, at which time the findings will be published. The findings will provide a detailed understanding of how the programme works and with whom it works best, as well as the different factors that may impact on family outcomes.

Open Access This article is distributed under the terms of the Creative Commons Attribution 4.0 International License (http:// creativecommons.org/licenses/by/4.0/), which permits unrestricted use, distribution, and reproduction in any medium, provided you give appropriate credit to the original author(s) and the source, provide a link to the Creative Commons license, and indicate if changes were made.
Publisher's Note Springer Nature remains neutral with regard to jurisdictional claims in published maps and institutional affiliations.

\section{References}

Achenbach, T. M., \& Edelbrock, C. S. (1983). Manual for the Child behavior checklist and revised Child behavior profile. Vermont: University of Vermont.

Ainsworth, M. D. S., \& Bell, S. M. (1970). Attachment, exploration, and separation: Illustrated by the behavior of one-year-olds in a strange situation. Child Development, 49-67.

Alderson, S., Westmarland, N., \& Kelly, L. (2013). The need for accountability to, and support for, children of men on domestic violence perpetrator programmes. Child Abuse Review, 22(3), 182-193.

Anderson, F., Howard, L., Dean, K., Moran, P., \& Khalifeh, H. (2016). Childhood maltreatment and adulthood domestic and sexual violence victimisation among people with severe mental illness. Social Psychiatry and Psychiatric Epidemiology, 51(7), 961-970.

Babor, F., Higgins-Biddle, J. C., Saunders, J. B., \& Monterio, M. G. (2008). The alcohol use disorders identification test - guidelines for use in primary care. Geneva: The World Health Orginisation.

Bair-Merritt, M. H., Blackstone, M., \& Feudtner, C. (2006). Physical health outcomes of childhood exposure to intimate partner violence: A systematic review. Pediatrics, 117(2), e278-e290.

Bakermans-Kranenburg, M. J., Van Ijzendoorn, M. H., \& Juffer, F. (2003). Less is more: Meta-analyses of sensitivity and attachment interventions in early childhood. Psychological Bulletin, 129(2), 195-215.

Barlow, J., \& Underdown, A. (2017). Child maltreatment during infancy: Atypical parent-infant relationships. Paediatrics and Child Health.

Bayley, N. (2006). Bayley scales of infant and toddler development: Bayley-III. Harcourt Assessment.

Beck, J. S. (2011). Cognitive behavior therapy: Basics and beyond: Guilford press.

Berman, A. H., Bergman, H., Palmstierna, T., \& Schylter, F. (2003). The drug use disorder identification test. Stockholm: Karolinska Institutet, Department of Clinical Neuroscience.

Berne, E. (2016). Transactional analysis in psychotherapy: A systematic individual and social psychiatry: Pickle Partners Publishing.

Bradshaw, J. (1992). Homecoming: Reclaiming and championing your inner child: Bantam.

Clements-Nolle, K., Wolden, M., \& Bargmann-Losche, J. (2009). Childhood trauma and risk for past and future suicide attempts among women in prison. Women's Health Issues, 19(3), 185-192.

Coid, J., Petruckevitch, A., Feder, G., Chung, W.-S., Richardson, J., \& Moorey, S. (2001). Relation between childhood sexual and physical abuse and risk of revictimisation in women: A cross-sectional survey. The Lancet, 358(9280), 450-454.

Constantino, R., Kim, Y., \& Crane, P. A. (2005). Effects of a social support intervention on health outcomes in residents of a domestic violence shelter: A pilot study. Issues in Mental Health Nursing, 26(6), 575-590.

Cox, J. L., Holden, J. M., \& Sagovsky, R. (1987). Detection of postnatal depression: Development of the 10-item Edinburgh postnatal depression scale. The British Journal of Psychiatry, 150(6), 782-786.

Crittenden, P. M. (1979). CARE-index: Coding manual. Unpublished manuscript, Miami, FL, 2004.

De Silva, M. J., Breuer, E., Lee, L., Asher, L., Chowdhary, N., Lund, C., \& Patel, V. (2014). Theory of change: A theory-driven approach to enhance the Medical Research Council's framework for complex interventions. Trials, 15(1), 267.

Devries, K. M., Child, J. C., Bacchus, L. J., Mak, J., Falder, G., Graham, $\mathrm{K}$., et al. (2014). Intimate partner violence victimization and alcohol consumption in women: A systematic review and meta-analysis. Addiction, 109(3), 379-391. 
Dube, S. R., Felitti, V. J., Dong, M., Chapman, D. P., Giles, W. H., \& Anda, R. F. (2003). Childhood abuse, neglect, and household dysfunction and the risk of illicit drug use: The adverse childhood experiences study. Pediatrics, 111(3), 564-572.

Edwards, V. J., Holden, G. W., Felitti, V. J., \& Anda, R. F. (2003). Relationship between multiple forms of childhood maltreatment and adult mental health in community respondents: Results from the adverse childhood experiences study. American Journal of Psychiatry, 160(8), 1453-1460.

Ehrensaft, M. K., Cohen, P., Brown, J., Smailes, E., Chen, H., \& Johnson, J. G. (2003). Intergenerational transmission of partner violence: A 20-year prospective study. Journal of Consulting and Clinical Psychology, 71(4), 741-753.

Ellsberg, M., \& Heise, L. (2002). Bearing witness: Ethics in domestic violence research. The Lancet, 359(9317), 1599-1604.

Evans, S. E., Davies, C., \& DiLillo, D. (2008). Exposure to domestic violence: A meta-analysis of child and adolescent outcomes. Aggression and Violent Behavior, 13(2), 131-140.

Felitti, V. J., Anda, R. F., Nordenberg, D., Williamson, D. F., Spitz, A. M., Edwards, V., \& Marks, J. S. (1998). Relationship of childhood abuse and household dysfunction to many of the leading causes of death in adults: The adverse childhood experiences (ACE) study. American Journal of Preventive Medicine, 14(4), 245-258.

Flach, C., Leese, M., Heron, J., Evans, J., Feder, G., Sharp, D., \& Howard, L. (2011). Antenatal domestic violence, maternal mental health and subsequent child behaviour: A cohort study. BJOG: An International Journal of Obstetrics \& Gynaecology, 118(11), 1383-1391.

Foa, E. B., Cashman, L., Jaycox, L., \& Perry, K. (1997). The validation of a self-report measure of posttraumatic stress disorder: The posttraumatic diagnostic scale. Psychological Assessment, 9, 445-451.

Gartstein, M. A., \& Rothbart, M. K. (2003). Studying infant temperament via the revised infant behavior questionnaire. Infant Behavior and Development, 26(1), 64-86.

Glover, V., \& Capron, L. (2017). Prenatal parenting. Current Opinion in Psychology, 15, 66-70.

Golding, J. M. (1999). Intimate partner violence as a risk factor for mental disorders: A meta-analysis. Journal of Family Violence, 14(2), 99-132.

Guss, S. S., Morris, A. S., Bosler, C., Castle, S. L., Hays-Grudo, J., Horm, D. M., \& Treat, A. (2018). Parents' adverse childhood experiences and current relationships with their young children: The role of executive function. Early Child Development and Care, 1-11.

Hamby, S. (2016). Self-report measures that do not produce gender parity in intimate partner violence: A multi-study investigation. Psychology of Violence, 6(2), 323-335.

Hegarty, K., Fracgp, Bush, R., \& Sheehan, M. (2005). The composite abuse scale: Further development and assessment of reliability and validity of a multidimensional partner abuse measure in clinical settings. Violence and Victims, 20(5), 529-547.

Heise, L. L. (1998). Violence against women: An integrated, ecological framework. Violence Against Women, 4(3), 262-290.

Hester, M., Ferrari, G., Jones, S., Williamson, E., Bacchus, L., Peters, T., \& Feder, G. (2015). Occurrence and impact of negative behaviour, including domestic violence and abuse, in men attending UK primary care health clinics: A cross-sectional survey. BMJ Open, 5(5), e007141.

Howard, L., Oram, S., Galley, H., Trevillion, K., \& Feder, G. (2013). Domestic violence and perinatal mental disorders: A systematic review and meta-analysis. PLoS Med, 10, e1001452. https://doi.org/ 10.1371/journal.pmed.1001452.

Hughes, K., Bellis, M. A., Hardcastle, K. A., Sethi, D., Butchart, A., Mikton, C., et al. (2017). The effect of multiple adverse childhood experiences on health: A systematic review and meta-analysis. The Lancet Public Health, 2(8), e356-e366.
Huth-Bocks, A. C., \& Hughes, H. M. (2008). Parenting stress, parenting behavior, and children's adjustment in families experiencing intimate partner violence. Journal of Family Violence, 23(4), 243-251.

Jack, S. M., Ford-Gilboe, M., Wathen, C. N., Davidov, D. M., McNaughton, D. B., Coben, J. H., et al. (2012). Development of a nurse home visitation intervention for intimate partner violence. BMC Health Services Research, 12(1), 1952.

Jahanfar, S., Howard, L. M., \& Medley, N. (2014). Interventions for preventing or reducing domestic violence against pregnant women. Cochrane Database of Systematic Reviews, 11, CD009414.

Johnson, V. K., \& Lieberman, A. F. (2007). Variations in behavior problems of preschoolers exposed to domestic violence: The role of mothers' attunement to children's emotional experiences. Journal of Family Violence, 22(5), 297-308.

Katz, E. (2015). Domestic violence, children's agency and mother-child relationships: Towards a more advanced model. Children \& Society, 29(1), 69-79.

Kellogg, S. (2014). Transformational chairwork: Using psychotherapeutic dialogues in clinical practice: Rowman \& Littlefield.

Kennedy, H., Landor, M., \& Todd, L. (2011). Video interaction guidance. London: Jessica Kingsley.

Kennedy, H., Ball, K., \& Barlow, J. (2017). How does video interaction guidance contribute to infant and parental mental health and wellbeing? Clinical Child Psychology and Psychiatry, 22(3), 500-517.

Khalifeh, H., Howard, L., Osborn, D., Moran, P., \& Johnson, S. (2013). Violence against people with disability in England and Wales: Findings from a national cross-sectional survey. PLoS One, 8(2), e55952.

Khalifeh, H., Moran, P., Borschmann, R., Dean, K., Hart, C., Hogg, J., et al. (2015). Domestic and sexual violence against patients with severe mental illness. Psychological Medicine, 45(4), 875-886.

Kitzmann, K. M., Gaylord, N. K., Holt, A. R., \& Kenny, E. D. (2003). Child witnesses to domestic violence: A meta-analytic review. Journal of Consulting and Clinical Psychology, 71(2), 339-352.

Labarre, M., Bourassa, C., Holden, G. W., Turcotte, P., \& Letourneau, N. (2016). Intervening with fathers in the context of intimate partner violence: An analysis of ten programs and suggestions for a research agenda. Journal of Child Custody, 13(1), 1-29.

Letourneau, N. L., Fedick, C. B., \& Willms, J. D. (2007). Mothering and domestic violence: A longitudinal analysis. Journal of Family Violence, 22(8), 649-659.

Levendosky, A. A., Huth-Bocks, A. C., Shapiro, D. L., \& Semel, M. A. (2003). The impact of domestic violence on the maternal-child relationship and preschool-age children's functioning. Journal of Family Psychology, 17(3), 275-287.

Lewis, G., Pelosi, A. J., Araya, R., \& Dunn, G. (1992). Measuring psychiatric disorder in the community: A standardized assessment for use by lay interviewers. Psychological Medicine, 22(2), 465-486.

Loseke, D. R., \& Kurz, D. (2005). Men's violence toward women is the serious social problem. Current Controversies on Family Violence, 2, 79-96.

Löwe, B., Decker, O., Müller, S., Brähler, E., Schellberg, D., Herzog, W., \& Herzberg, P. Y. (2008). Validation and standardization of the generalized anxiety disorder screener (GAD-7) in the general population. Medical Care, 46(3), 266-274.

Lucassen, N., Tharner, A., Van IJzendoorn, M. H., BakermansKranenburg, M. J., Volling, B. L., Verhulst, F. C., et al. (2011). The association between paternal sensitivity and infant-father attachment security: A meta-analysis of three decades of research. Journal of Family Psychology, 25(6), 986-992.

Lyons Ruth, K., \& Block, D. (1996). The disturbed caregiving system: Relations among childhood trauma, maternal caregiving, and infant affect and attachment. Infant Mental Health Journal, 17(3), 257-275.

Machisa, M. T., Christofides, N., \& Jewkes, R. (2016). Structural pathways between child abuse, poor mental health outcomes 
and male-perpetrated intimate partner violence (IPV). PLoS One, 11(3), e0150986.

MacMillan, H. L., Wathen, C. N., Jamieson, E., Boyle, M. H., Shannon, H. S., Ford-Gilboe, M., et al. (2009). Screening for intimate partner violence in health care settings: A randomized trial. JAMA, 302(5), 493-501.

Malmberg, L. E., Lewis, S., West, A., Murray, E., Sylva, K., \& Stein, A. (2016). The influence of mothers' and fathers' sensitivity in the first year of life on children's cognitive outcomes at 18 and 36 months. Child: Care, Health and Development, 42(1), 1-7.

Margolin, G., \& Gordis, E. B. (2003). Co-occurrence between marital aggression and parents' child abuse potential: The impact of cumulative stress. Violence and Victims, 18(3), 243-258.

McConnell, N., Barnard, M., \& Taylor, J. (2017). Caring dads safer children: Families' perspectives on an intervention for maltreating fathers. Psychology of Violence, 7(3), 406-416.

Meyer, S. (2018). Motivating perpetrators of domestic and family violence to engage in behaviour change: The role of fatherhood. Child \& Family Social Work, 23(1), 97-104.

Moore, T., Arefadib, N., Deery, A., Keyes, M., \& West, S. (2017). The First Thousand Days: An Evidence Paper. Retrieved from

Moran, P., Leese, M., Lee, T., Walters, P., Thornicroft, G., \& Mann, A. (2003). Standardised assessment of personality - abbreviated scale (SAPAS): Preliminary validation of a brief screen for personality disorder. The British Journal of Psychiatry, 183, 228-232.

Murray, L., \& Cooper, P. J. (1996). The impact of postpartum depression on child development. International Review of Psychiatry, 8(1), 55-63.

Murray, L., Fearon, P., \& Cooper, P. (2015). Postnatal depression, mother-infant interactions, and child development. Identifying perinatal depression and anxiety: Evidence-based practice in screening, psychosocial assessment, and management, 139-164.

Norman, R. E., Byambaa, M., De, R., Butchart, A., Scott, J., \& Vos, T. (2012). The long-term health consequences of child physical abuse, emotional abuse, and neglect: A systematic review and meta-analysis. PLoS Medicine, 9(11), e1001349.

NSCDC. (2007). The Timing and Quality of Early Experiences Combine to Shape Brain Architecture: Working Paper No. 5. Retrieved from.

Nugent, J. K. (2015). The newborn behavioral observations (NBO) system as a form of intervention and support for new parents. Zero to Three Journal, 36(1), 2-10.

Nusslock, R., \& Miller, G. E. (2016). Early-life adversity and physical and emotional health across the lifespan: A neuroimmune network hypothesis. Biological Psychiatry, 80(1), 23-32.

O'Cathain, A., Murphy, E., \& Nicholl, J. (2007). Why, and how, mixed methods research is undertaken in health services research in England: A mixed methods study. BMC Health Services Research, 7(1), 85.

Office for National Statistics. (2016). National Records of Scotland ; Northern Ireland Statistics and Research Agency (2016): 2011 Census aggregate data.

Office for National Statistics. (2018). Population estimates for the UK, England and Wales, Scotland and Northern Ireland: mid-2017.

Överlien, C. (2017). 'Do you want to do some arm wrestling?': children's strategies when experiencing domestic violence and the meaning of age. Child \& Family Social Work, 22(2), 680-688.

Radford, L., Aitken, R., Miller, P., Ellis, J., Roberts, J., \& Firkic, A. (2011). Meeting the needs of children living with domestic violence in London: Research report. Retrieved from London:

Reuben, A., Moffitt, T. E., Caspi, A., Belsky, D. W., Harrington, H., Schroeder, F., et al. (2016). Lest we forget: Comparing retrospective and prospective assessments of adverse childhood experiences in the prediction of adult health. Journal of Child Psychology and Psychiatry, 57(10), 1103-1112.

Riggs, D. S., Caulfield, M. B., \& Street, A. E. (2000). Risk for domestic violence: Factors associated with perpetration and victimization. Journal of Clinical Psychology, 56(10), 1289-1316.

Rollnick, S., \& Miller, W. R. (1995). What is motivational interviewing? Behavioural and Cognitive Psychotherapy, 23(4), 325-334.

Rothman, E. F., Mandel, D. G., \& Silverman, J. G. (2007). Abusers' perceptions of the effect of their intimate partner violence on children. Violence Against Women, 13(11), 1179-1191.

Salisbury, E. J., Henning, K., \& Holdford, R. (2009). Fathering by partner-abusive men: Attitudes on children's exposure to interparental conflict and risk factors for child abuse. Child Maltreatment, 14(3), 232-242.

Schumacher, J. A., Coffey, S. F., Stasiewicz, P. R., Murphy, C. M., Leonard, K. E., \& Fals-Stewart, W. (2011). Development of a brief motivational enhancement intervention for intimate partner violence in alcohol treatment settings. Journal of Aggression, Maltreatment \& Trauma, 20(2), 103-127.

Stanley, N. (2011). Children experiencing domestic violence: a research review: Research in practice Dartington.

Stanley, N., \& Humphreys, C. (2017). Identifying the key components of a'whole family'intervention for families experiencing domestic violence and abuse. Journal of Gender-Based Violence, 1(1), 99-115.

Stanley, N., Fell, B., Miller, P., Thomson, G., \& Watson, J. (2012a). Men's talk: men's understandings of violence against women and motivations for change. Violence Against Women, 18(11), $1300-1318$.

Stanley, N., Graham Kevan, N., \& Borthwick, R. (2012b). Fathers and domestic violence: Building motivation for change through perpetrator programmes. Child Abuse Review, 21(4), 264-274.

Stein, A., Pearson, R. M., Goodman, S. H., Rapa, E., Rahman, A., McCallum, M., et al. (2014). Effects of perinatal mental disorders on the fetus and child. The Lancet, 384(9956), 1800-1819.

Stover, C. S., Easton, C. J., \& McMahon, T. J. (2013). Parenting of men with co-occurring intimate partner violence and substance abuse. Journal of Interpersonal Violence, 28(11), 2290-2314.

Sun, J., Patel, F., Rose-Jacobs, R., Frank, D. A., Black, M. M., \& Chilton, M. (2017). Mothers' adverse childhood experiences and their young Children's development. American Journal of Preventive Medicine, 53(6), 882-891.

Trevillion, K., Oram, S., Feder, G., \& Howard, L. M. (2012). Experiences of domestic violence and mental disorders: A systematic review and meta-analysis. PLoS One, 7(12), e51740.

Trevillion, K., Byford, S., Cary, M., Rose, D., Oram, S., Feder, G., et al. (2013). Linking abuse and recovery through advocacy: An observational study. Epidemiology and Psychiatric Sciences, 30, 1-15.

Van der Kolk, B. A. (2015). The body keeps the score: Brain, mind, and body in the healing of trauma: Penguin Books.

Van der Kolk, B. A. (2017). Developmental trauma disorder: Toward a rational diagnosis for children with complex trauma histories. Psychiatric Annals, 35(5), 401-408.

Whitaker, R. C., Dearth-Wesley, T., Gooze, R. A., Becker, B. D., Gallagher, K. C., \& McEwen, B. S. (2014). Adverse childhood experiences, dispositional mindfulness, and adult health. Preventive Medicine, 67, 147-153.

WHO. (2001). Putting women first: ethical and safety recommendations for research on domestic violence against women. 